\title{
FARMACOGENÉTICA DE FÁRMACOS HIPOLIPEMIANTES
}

\author{
PHARMACOGENETIC OF LIPID-LOWERING DRUGS
}

Marilu Fiegenbaum ${ }^{1}$, Mara H. Hutz ${ }^{2}$

\begin{abstract}
${ }^{1}$ Docente. Centro Universitário Metodista. Instituto Porto Alegre. ${ }^{2}$ Docente. Departamento de Genética. Instituto de Biociências UFRGS.

Correspondência: Profa. Dra. Mara H. Hutz - Av. Bento Gonçalves, 9500

Caixa Postal 15053 - Bairro Agronomia - Porto Alegre - RS / CEP: 91501-970

Fone: +55 5133166722 / e-mail: mara.hutz@ufrgs.br
\end{abstract}

Fiegenbaum M, Hutz MH. Farmacogenética de fármacos hipolipemiantes. Medicina (Ribeirão Preto) 2006; 39 (4): 543-53.

RESUMO: Embora o tratamento farmacoterapêutico para redução dos níveis de colesterol tenha sido um dos grandes avanços no tratamento de doenças cardiovasculares e aterosclerose, os benefícios são ainda limitados devido a variabilidade interindividual na resposta a esses medicamentos. Entre os fatores importantes para a avaliação da variabilidade interindividual pode-se citar a severidade da doença, adesão ao tratamento, condições fisiológicas, condições biológicas e o perfil genético do paciente. Neste contexto, três grandes grupos de genes podem ser analisados: a) Genes que codificam proteínas envolvidas na metabolização e/ou transporte dos fármacos, influenciando a farmacocinética dos compostos. b) Genes que codificam proteínas envolvidas no mecanismo de ação e/ou nas rotas metabólicas em que o fármaco age (farmacodinâmica). c) Genes que codificam proteínas envolvidas no desenvolvimento direto da doença ou de fenótipos intermediários. Nessa revisão discutimos os estudos farmacogenéticos dos principais fármacos hipolipemiantes e a expectativa em relação a fármacogenética de auxiliar em nossa capacidade de predição em relação à eficácia do tratamento e a possibilidade de redução dos efeitos adversos a esses medicamentos. Na medida que novos estudos forem efetuados e que a grande parte da variabilidade genética envolvidas nas diferenças interindividuais na ação de fármacos for elucidada, o grande desafio será a aplicação desses conhecimentos na prática médica.

Descritores: Hipolipemiantes. Farmacogenética. Variação (Genética). Doenças Cardiovasculares.

\section{1- INTRODUÇÃO}

Nas últimas décadas, a pesquisa na área da saúde tem contribuído de forma considerável para a redução da mortalidade por doenças cardiovasculares ${ }^{1,2}$. A redução de mortalidade observada é decorrente, em parte, de uma melhor compreensão dos principais fatores de risco modificáveis para a doença cardiovascular (como tabagismo, hipertensão, dislipidemias e diabete mellitus) e de intervenções farmacoterapêuticas para cada um destes fatores de risco.

Dentre os fatores citados, níveis elevados de colesterol, especialmente de lipoproteínas de baixa densidade (LDL), bem como de triglicerídeos e baixos níveis de lipoproteínas de alta densidade (HDL) constituem os principais alvos de tratamento segundo as diretrizes brasileiras ${ }^{3}$ e americanas ${ }^{4}$ para o controle de doenças do coração. Estas diretrizes preconi- 
zam a utilização de fármacos hipolipemiantes de acordo com o risco individual de cada paciente desenvolver problemas cardiovasculares (prevenção primária) ou de desenvolver um novo evento cardiovascular (prevenção secundária).

A farmacoterapêutica para quadros de dislipidemia se baseia em diversas classes de fármacos como resinas seqüestrantes de ácidos biliares, fibratos, ácido nicotínico, inibidores seletivos da absorção de colesterol e inibidores da 3-hidroxi 3-metilglutaril coenzima-A (HMG Coa) redutase (estatinas ou vastatinas).

Embora o tratamento farmacoterapêutico para redução dos níveis de colesterol tenha sido um dos grandes avanços no tratamento de doenças cardiovasculares e aterosclerose, a variabilidade interindividual na resposta é um dos fatores que limita o benefício alcançado. Entre os fatores importantes para a avaliação da variabilidade interindividual pode-se citar a severidade da doença, adesão ao tratamento, condições fisiológicas, condições biológicas e o perfil genético do paciente. Em estudos randomizados realizados com fármacos hipolipemiantes observam-se vários subgrupos em que a resposta é diferenciada. No estudo CARE, por exemplo, a redução de eventos cardiovasculares com o uso de pravastatina foi mais acentuada em mulheres (-46\% [95CI\% -22 a -62\%]) do que em homens $(-20 \%$ [95CI\% -8 a $-30 \%])^{5}$. No estudo $4 \mathrm{~S}$, a redução de eventos cardiovasculares com tratamento com sinvastatina foi inversamente associada aos valores basais (pré-tratamento) de colesterol dos participantes, sugerindo que aqueles que apresentam uma síntese maior de colesterol endógeno respondem melhor do que aqueles que apresentam uma síntese menor ${ }^{6}$. Recentemente, inúmeros trabalhos também têm evidenciado a importância de polimorfismos genéticos para a eficácia (redução dos níveis lipídicos e/ou prevenção de eventos cardíacos) e predição de efeitos adversos (revisões $\mathrm{em}^{7,8}$ ). Neste contexto, três grandes grupos de genes candidatos podem ser analisados:

1) Genes que codificam proteínas envolvidas na metabolização e/ou transporte dos fármacos, influenciando a farmacocinética dos compostos.

2) Genes que codificam proteínas envolvidas no mecanismo de ação e/ou nos rotas metabólicas em que o fármaco age (farmacodinâmica)

3) Genes que codificam proteínas envolvidas no desenvolvimento direto da doença ou de fenótipos intermediários.

\section{2- FARMACOGENÉTICA DE FÁRMACOS HIPOLIPEMIANTES}

\section{1- Os inibidores da HMG Coa redutase}

\subsubsection{Mecanismo de ação e efeito hipolipe- miante}

Os inibidores da 3-hidroxi 3-metilglutaril Coenzima A (estatinas ou vastatinas) são fármacos que causam a inibição parcial e reversível da enzima HMG Coa redutase, enzima chave na rota da síntese do colesterol endógeno. Os inibidores da HMG Coa redutase possuem uma porção similar ao ácido mevalônico, inibindo a conversão de HMG-Coa em mevalonato9 . Em humanos, a biossíntese de colesterol a partir de acetilCoa fornece cerca de 60 a $70 \%$ do colesterol circulante, o que faz com que a inibição da via sintética seja uma forma bastante eficiente de terapia hipolipemiante.

A redução de colesterol intracelular promove um aumento da expressão dos receptores de LDL (LDL-R) na superfície dos hepatócitos e resulta em uma maior recaptação de LDL circulantes. Como um mecanismo hipolipemiante adicional, os inibidores da HMG Coa redutase reduzem os níveis de lipoproteínas de muito baixa densidade (VLDL) por inibição da sua síntese e promoção de seu catabolismo. As VLDLs são precursores das LDLs e a inibição de sua produção resulta em uma redução adicional dos níveis de LDL. A inibição da formação de VLDLs é secundária a inibição da síntese do colesterol, necessário para a formação da lipoproteína, e pela redução dos níveis de apolipoproteína B, a principal apolipoproteína presente em LDLs ${ }^{10}$.

Atualmente seis estatinas estão no mercado: atorvastatina, fluvastatina, lovastatina, pravastatina, rosuvastatina e sinvastatina. Dados dos maiores estudos randomizados envolvendo estes fármacos mostraram que as estatinas são as mais eficientes em baixar os níveis de colesterol total (18 a 25\%) e LDLcolesterol (25 a 55\%). Além disso, também reduzem moderadamente os níveis de triglicerídeos (10 a 15\%) e aumentam da mesma forma os níveis de HDL-colesterol (5 a 10\%). Estes estudos também mostraram que a utilização de estatinas diminui os riscos de eventos cardíacos fatais e não fatais independentemente de outros fatores como a idade, sexo, tabagismo, diabetes ou hipertensão ${ }^{11 / 14}$. 


\subsubsection{Genes envolvidos na farmacodinâmica e/ou no desenvolvimento direto da doença ou de fenótipos intermediários}

Inúmeros genes relacionados à farmacodinâmica e com fenótipos intermediários da doença car- diovascular já foram analisados quanto a sua contribuição para a variabilidade de resposta observada ao tratamento com estatinas. A Tabela I apresenta o resultado de alguns dos trabalhos nesta área.

No grupo de genes relacionados ao metabolismo de lipídeos, um dos genes mais estudados é o que

Tabela I: Exemplos de trabalhos publicados sobre farmacogenética de estatinas.

\begin{tabular}{|c|c|c|c|c|}
\hline Genes & SNP & $\begin{array}{c}\text { № de } \\
\text { pacientes }\end{array}$ & Resultados & eferência \\
\hline APOE & E2, E3, E4 & 145 & $\begin{array}{l}\text { Redução LDL } E^{\star} 3 / E^{\star} 3>E^{*} 4 \text {, } \\
\text { aumento } H D L E^{\star} 2>E^{\star} 3 / E^{\star} 3=E^{\star} 4\end{array}$ & 18 \\
\hline APOE & $\mathrm{E} 2, \mathrm{E} 3, \mathrm{E} 4$ & 328 & $\begin{array}{l}\text { Redução LDL e TG E*2 > E*3/E*3 }=E^{\star} 4 \\
\text { (só em homens) }\end{array}$ & 19 \\
\hline APOE & $\mathrm{E} 2, \mathrm{E} 3, \mathrm{E} 4$ & 401 & SNP não associados à resposta & 21 \\
\hline CETP & TaqIB & 411 & $\begin{array}{l}\text { SNP associado à variabilidade de } \\
\text { redução de eventos cardíacos }\end{array}$ & 26 \\
\hline CETP & TaqIB & 1606 & $\begin{array}{l}\text { SNP não associado à variabilidade } \\
\text { de redução de eventos cardíacos }\end{array}$ & 67 \\
\hline CETP & TaqIB & 217 & $\begin{array}{l}\text { SNP associado à variabilidade } \\
\text { de aumento de HDL }\end{array}$ & 30 \\
\hline CETP & TaqIB & 720 & $\begin{array}{l}\text { SNP associado à variabilidade } \\
\text { de redução de eventos cardíacos }\end{array}$ & 28 \\
\hline CETP & 9 SNPs & 98 & $\begin{array}{l}\text { Haplótipos associados à resposta } \\
\text { de HDL e triglicerídeos }\end{array}$ & 29 \\
\hline SCAP, SREBF-1a & 2 SNPs & 191 & $\begin{array}{l}\text { SNP -36del/G (SREBF-1a) associado } \\
\text { diferenças de aumento de apoA-I }\end{array}$ & 68 \\
\hline APOE, CETP, LIPC & 3 SNPs & 99 & $\begin{array}{l}\text { SNP TaqIB (CETP) associado } \\
\text { a resposta de HDL }\end{array}$ & 23 \\
\hline SCAP, SREBF-1a, SREBF-2 & 3 SNPs & 99 & $\begin{array}{l}\text { SNP lle796Val (SCAP) asociado a } \\
\text { resposta de colesterol total }\end{array}$ & 33 \\
\hline $\begin{array}{l}\text { CYP3A4, CYP3A5, } \\
\text { ABCB1 }\end{array}$ & 5 SNPs & 116 & $\begin{array}{l}\text { SNPs } 1236 \mathrm{C} / \mathrm{T}, 2677 \mathrm{G} / \mathrm{AT} \text { e } 3435 \mathrm{C} / \mathrm{T} \\
\text { associados à eficácia e desenvolviment } \\
\text { de mialgia }\end{array}$ & to \\
\hline CYP3A4 & 3 SNPs & 340 & SNP $-290 \mathrm{~A} / \mathrm{G}$ associado a resposta & 37 \\
\hline ABCG5, ABCG8 e CYP7A1 & 6 SNPs & 337 & $\begin{array}{l}\text { Interação dos SNPs D19H (ABCG8) } \\
\text { e -204A/C (CYP7A1) na resposta }\end{array}$ & 34 \\
\hline $\mathrm{ABCB} 1$ & 2 SNPs & 344 & Haplótipos associados à resposta & 44 \\
\hline ABCB1 & 2 SNPs & 69 & SNPs não associados a resposta & 46 \\
\hline $\begin{array}{l}\text { ABCG5, ABCG8, APOB, } \\
\text { APOE, CETP, CYP3A4, } \\
\text { CYP3A5, FDFT1, } \\
\text { HMGCR, LDLR }\end{array}$ & 148 SNPs & 1536 & $\begin{array}{l}2 \text { SNPs de intron do gene HMGCR } \\
\text { associados a resposta }\end{array}$ & 22 \\
\hline
\end{tabular}

SNP: polimorfismo de base única. 
codifica a apolipoproteína $\mathrm{E}(A P O E)$. A apolipoproteína $\mathrm{E}$ tem um papel fundamental no metabolismo lipídico, sendo encontrada em várias partículas lipoprotéicas (quilomícrons, VLDLs e remanescentes, HDLs), onde atua como ligante para diversos receptores (LDL-R e LRP principalmente). O gene da apo E está localizado no braço longo do cromossomo 19 juntamente com as apos C-I, C-II e C-IV. O loco é polimórfico e apresenta três alelos comuns $\left(E^{*} 2, E^{*} 3\right.$ e $\left.E^{* 4}\right)$ que codificam três isoformas (E2, E3 e E4) que diferem nos resíduos 112 e/ou 158 da proteína. A isoforma $\mathrm{E} 3$, mais comum, possui uma arginina na posição 158 e uma cisteína na posição 112. As isoformas E2 e E4 possuem cisteína e arginina nas duas posições, respectivamente ${ }^{15}$. O polimorfismo da apo $\mathrm{E}$ afeta a concentração de LDL modulando a expressão do receptor de LDL. Com uma maior afinidade pelos receptores, a isoforma $\mathrm{E} 4$ aumenta o catabolismo de lipoproteínas remanescentes, o que diminui o número de receptores de LDL hepáticos e aumenta os níveis de LDL circulantes. $\mathrm{O}$ alelo $E^{* 2} 2$ tem um efeito contrário sobre os níveis de LDL, uma vez que sua afinidade pelo receptor é menor e desta maneira há um aumento de receptores de $\mathrm{LDL}^{16}$. Em relação à farmacogenética, o efeito destas variantes sobre a variabilidade de resposta das estatinas foi investigado em diversos estudos. Alguns estudos demonstraram que portadores do alelo $E^{*} 2$ se beneficiam mais com o tratamento com estes fármacos do que portadores do alelo $E^{*} 4$ e homozigotos $E^{*} 3 / E^{*} 3^{17 / 19}$. No entanto, estes resultados não foram confirmados por outros estudos $^{20 / 23}$.

Outro gene bastante estudado é o da proteína transferidora de ésteres de colesterol (CETP). A CETP é a proteína que promove a troca de lipídeos entre as partículas lipoprotéicas, transferindo ésteres de colesterol das HDLs para outras lipoproteínas para subseqüente recaptação pelo fígado. Por aumentar o conteúdo de ésteres de colesterol de LDLs e VLDLs, a CETP aumenta a aterogenecidade destas lipoproteínas. Vários polimorfismos no gene da CETP foram descritos, mas um em especial, referido como TaqIB, localizado no $227^{\circ}$ nucleotídeo do primeiro intron está associado com a atividade transferidora de ésteres de colesterol $^{24}$ e concentrações de HDL-colesterol ${ }^{24 / 26}$. Em uma meta-análise realizada envolvendo mais de 10.000 indivíduos, este polimorfismo foi significantemente associado com níveis de HDL e CETP ${ }^{27}$. Recentemente, vários estudos têm demonstrado que a variante TaqIB está associada a variabilidade de res- posta (Tabela I). Em pacientes tratados com estatinas, o polimorfismo TaqIB foi associado a riscos diferenciados de eventos cardiovasculares ${ }^{26,28}$ e variabilidade de aumento dos níveis de HDL-c 23,29,30.

Outra rota promissora desta área da farmacogenética está relacionada à rota de síntese de colesterol e seu controle via fatores de transcrição denominados sterol regulatory element-binding factors (SREBFs) e pela proteína SCAP (cleavage-activating protein), responsável pela ativação dos SREBFs. A lista de genes regulados pela rota SCAP-SREBF inclui inúmeros genes envolvidos na síntese e recaptação de colesterol, triglicerídeos, ácidos graxos, inclusive o próprio gene $H M G C R$, que codifica a 3-hidroxi3metilglutaril Coenzima A redutase, alvo das estatinas $^{31,32}$. Fiegenbaum et al. ${ }^{33}$ demonstraram que, em uma amostra de brasileiros descendentes de europeus tratados com sinvastatina $20 \mathrm{mg}$, a variante $2386 \mathrm{~A}>\mathrm{G}$ do gene SCAP foi significantemente associada à redução dos níveis de colesterol total. Enquanto $61 \%$ dos portadores do alelo $2386 \mathrm{G}$ obtiveram uma resposta superior a media geral $(-27,8 \%)$ somente $29 \%$ dos homozigotos alcançaram tal redução (Figura 1).

O gene $H M G C R$ foi recentemente investigado por Chasman et al ${ }^{22}$ em uma amostra composta por 1536 indivíduos. Esses autores analisaram a influência de 148 polimorfismos em 10 genes candidatos incluindo dois polimorfismos no gene $H M G C R$. Essas duas variantes estavam associadas com a resposta do tratamento com pravastatina $40 \mathrm{mg} / \mathrm{dia}$. Outro estudo demonstrou que polimorfismos dos genes CYP7A1, que codifica a colesterol $7 \alpha$-hidroxilase, enzima-chave na rota de síntese de ácidos biliares e ABCG5/G8, que codificam proteínas responsáveis pelo transporte de colesterol do fígado para a bile, interagem de maneira dose dependente na resposta ao tratamento com atorvastatina $10 \mathrm{mg}^{34}$.

\subsubsection{Genes que codificam proteínas envolvidas na metabolização e/ou transporte dos fár- macos}

Embora o mecanismo de ação dos inibidores da HMG Coa redutase seja semelhante, a biotransformação das estatinas varia consideravelmente. A atorvastatina, a cerivastatina (retirada do mercado nacional em 2001), a lovastatina e a sinvastatina são substratos do citocromo $\mathrm{P} 450$ 3A4/3A5. A pravastatina não é extensivamente metabolizada via citocromo P450, enquanto a fluvastatina é metabolizada pelo CYP2C $9^{35}$. 


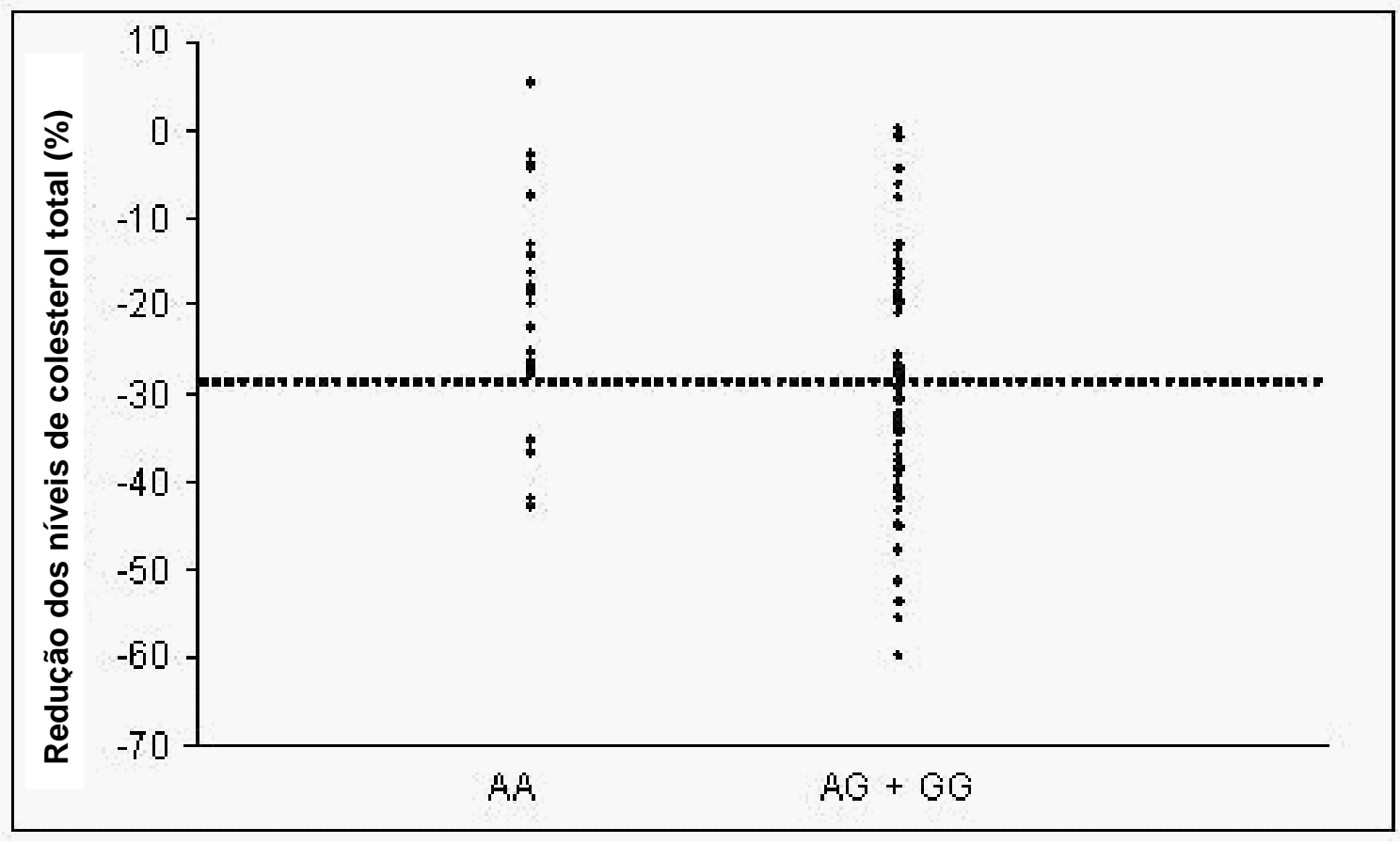

Figura 1: Variação de níveis de colesterol total com o tratamento com sinvastatina em pacientes com diferentes genótipos do gene SCAP. A linha representa a média de redução em todos os pacientes (-27,8\%). Em portadores do alelo $\mathrm{G} 60 \%$ dos pacientes apresentam uma redução maior que a média, enquanto somente $29 \%$ do homozigotos AA apresentam tal redução (P 0,009). Adaptado de Fiegenbaum et al (Pharmacogenomics J 2005; 5: 359-364) ${ }^{33}$.

A isoenzima CYP3A4 é a forma mais abundante entre as enzimas da superfamília citocromo P450, metabolizando cerca de $50 \%$ dos fármacos. Embora exista uma grande variabilidade interindividual na expressão desta isoforma de CYP, não existem evidências de alelos nulos para este gene e esta variabilidade provavelmente se deva a grande variedade de xenobióticos indutores e inibidores desta enzima e de variantes genéticas ao longo do gene. Dos polimorfismos já descritos, o mais comum é uma transição $A>G$ localizada na posição -392 (CYP3A4*1B), em uma seqüência conhecida como nifedipine-specific element. O gene CYP3A5 é expresso somente em $5-15 \%$ de indivíduos de ancestralidade européia e em $45-73 \%$ de ancestralidade européia ${ }^{36}$. O alelo CYP3A5*3 (6986A>G) é o mais freqüentemente associado com a não expressão do gene nestes grupos ${ }^{36}$.

A influência desta variante sobre o efeito hipolipemiante da atorvastatina foi avaliada por Kajinami et al. ${ }^{37}$. Neste trabalho, o alelo CYP $3 \mathrm{~A} 4 * 1 \mathrm{~B}$ foi associado com os níveis de LDL pós-tratamento, mas não com a variação de resposta em si. O alelo CYP3A5*3 foi associado à variabilidade de resposta em pacientes que utilizam estatinas metabolizadas por
CYP3A4/A5 ${ }^{38}$. Estes resultados, no entanto não foram observados em outros estudos ${ }^{39}$.

Historicamente o metabolismo é visto como o principal determinante da biodisponibilidade de fármacos. Recentemente, no entanto, evidências claras têm demonstrado que transportadores de membrana também têm importância crítica. Recentes estudos in vitro mostraram que algumas estatinas (sinvastatina, atorvastatina e pravastatina, por exemplo) também atuam como substratos de transportadores de membrana $^{40}$.

As proteínas transportadoras de fármacos podem ser separadas em duas grandes classes: transportadores de efluxo e transportadores de captação. Transportadores de captação facilitam o transporte de fármacos para o interior das células. Nesta classe estão inclusas as famílias dos polipeptídeos transportadores de anions orgânicos (OATP), dos transportadores de anion orgânicos (OAT), dos transportadores de cátions orgânicos (OCT), dos transportadores de cátions/carnitina orgânicos (OCTN) e dos transportadores peptídicos (PEPT). Os transportadores de efluxo, no entanto, agem exportando fármacos e substâncias do interior da célula para o meio extracelular, muitas ve- 
zes contra o gradiente de concentração. A maioria dos transportadores de efluxo é membro da superfamília da adenosine triphosphate (ATP)-binding cassete $(A B C)$. Fazem parte desta classe de transportadores a glicoproteína-P (também conhecida como MDR1), a bomba de efluxo de sais biliares (BSEP), proteína relacionada ao MDR (MRP) e a proteína de resistência do câncer de mama (BCRP) ${ }^{40}$.

A glicoproteína-P (Pg-P), codificada pelo gene $A B C B 1$ (ATP-binding cassete, subfamily B) foi primeiramente descrita em células neoplásicas como a proteína responsável pela resistência ao tratamento com quimioterápicos. A Pg-P também está presente em tecidos normais, onde desempenha funções de defesa contra substâncias potencialmente tóxicas. O gene $A B C B 1$, mapeado no cromossomo $7 \mathrm{q} 21.1$, foi extensivamente estudado para identificação de variantes funcionais. O estudo de maior impacto, conduzido por Hoffmeyer et al. ${ }^{41}$ descreveu 15 polimorfismos ao longo do gene $A B C B 1$. Dentre estes, o polimorfismo $C 3435 T$ parece ter efeitos farmacológicos e estar associado com a expressão da Pg-P no intestino, influenciando o transporte e a biodisponibilidade de substratos desta proteína. $\mathrm{O}$ alelo $\mathrm{T}$, particularmente em homozigose, está associado com diminuição nos níveis de expressão da Pg-P. A freqüência deste genótipo em populações caucasóides é de 24-29\% ${ }^{41,42,43}$. Este polimorfismo é uma troca sinônima no éxon 26 e está em desequilíbrio de ligação com outros SNPs não sinônimos ao longo do gene, especialmente $1236 \mathrm{C}>\mathrm{T}$ e $2677 \mathrm{G}>\mathrm{A} / \mathrm{T}$.

Kajinami et al. ${ }^{44}$ descreveram um estudo farmacogenético dos polimorfismos $2677 \mathrm{G}>\mathrm{A} / \mathrm{T}$ e $3435 \mathrm{C}>\mathrm{T}$ e o tratamento com atorvastatina $10 \mathrm{mg}$. Os resultados sugerem que portadores do alelo $3435 \mathrm{~T}$ têm uma redução maior dos níveis de LDL quando comparados aos homozigotos $3435 \mathrm{CC}$. Estes resultados foram corroborados por Fiegenbaum et al. ${ }^{39}$, que observaram resultados semelhantes em uma amostra de brasileiros descendentes de europeus tratados com sinvastatina $20 \mathrm{mg}$, também substrato da Pg-P. Estes resultados, no entanto, não foram confirmados por Thompson et al. ${ }^{45}$ e por Rodrigues et al. ${ }^{46}$.

Algumas estatinas (pravastatina, rosuvastatina e cerivastatina) também são substratos para a proteína de transporte de captação OATP. A proteína OATP$\mathrm{C}$ ou OATP1B1 (codificada pelo gene $S L C O 1 B 1$ ou $S L C 21 A 6)$ é expressa predominantemente na membrana basolateral dos hepatócitos ${ }^{47,48}$, o que indica que sua função é captar substratos da circulação portal e facilitar sua excreção ${ }^{49,50}$. A OATP-C apresenta vários polimorfismos comuns e sua relação com a eficácia das estatinas ainda é incerta. Das estatinas, a pravastatina, a rosuvastatina e a cerivastatina são ativamente transportadas por este mecanismo. Em dois estudos farmacocinéticos realizado com pravastatina, foram observados que diferentes haplótipos do gene SLCO1B1 apresentam diferentes capacidades de transporte do fármaco, o que pode se refletir em variabilidade interindividual de resposta ${ }^{49,51}$.

\subsubsection{Farmacogenética versus efeitos adversos}

Como uma classe de medicamentos, as estatinas são bem toleradas e raramente efeitos adversos graves são observados. Os efeitos adversos comuns incluem distúrbios gastrintestinais, dispepsia, dores de cabeça, mialgia, distúrbios no sistema nervoso central e desordens do sono. Hepatotoxicidade e anormalidades musculares são os efeitos adversos clinicamente mais importantes. Aumento das concentrações de transaminases hepáticas (transaminase oxalacética - TGO e transaminase pirúvica - TGP) e creatina fosfoquinase (CK ou CPK) a mais de três vezes o limite superior do normal foram descritos em 3 a 5\% dos pacientes. Entretanto, a elevação destas enzimas geralmente é temporária e os pacientes permanecem assintomáticos. Anormalidades musculares podem começar com uma mialgia e evoluir até miopatia, (elevação de CK acima de 10 vezes o valor normal) ou rabdomiólise, onde além do aumento de CK ocorre mioglobinemia, mioglobinúria e necrose muscular extensiva. Quando as estatinas são prescritas como monoterapia, a incidência de miopatia situa-se entre $0,1-0,5 \%$ dos casos e é dose relacionada ${ }^{52,53}$. Dados da International Drug Information System (INTDIS) relatam uma frequiência de 7,6 a $14,4 \%$ para mialgia, 0,2 a $1,8 \%$ para miopatia e 0,2 a $2,1 \%$ para rabdomiólise em pacientes que utilizaram diferentes estatinas ${ }^{54}$.

O mecanismo preciso pelo qual as estatinas induzem desordens musculares ainda não está estabelecido, mas os fatores de risco incluem:

1) altas doses de estatinas;

2) baixos níveis intestinais e hepáticos das enzimas envolvidas na metabolização, o que eleva os níveis circulantes do fármaco;

3) co-administração de inibidores das enzimas de metabolização, o que também aumenta os níveis de estatina circulantes ${ }^{54}$. 
Embora esta área da farmacogenética seja de grande interesse, os estudos relacionando polimorfismos em genes de metabolização e/ou transporte e o desenvolvimento de efeitos adversos ainda são incipientes. Até o momento os genes CYP3A4 e ABCB1 foram associados ao desenvolvimento de efeitos adversos. No primeiro gene, foi demonstrado que embora a variante CYP3A4*1B não esteja associada ao risco de desenvolvimento de efeitos adversos musculares, a severidade do dano muscular induzido por atorvastatina está relacionada aos genótipos de CYP3A4 ${ }^{55}$. Já para o gene ABCB1 as variantes 1236T, 2677A/T e $3435 \mathrm{~T}$ foram menos comuns em indivíduos com mialgia em um estudo com sinvastatina ${ }^{39}$.

\section{2- Fibratos}

\subsubsection{Mecanismo de ação e efeito hipolipemiante}

Os fibratos são fármacos hipolipemiantes que reduzem os níveis de triglicerídeos e colesterol. Os efeitos mais pronunciados dos fibratos são relacionados à diminuição dos níveis de lipoproteínas ricas em triglicerídeos e das subfrações mais aterogênicas de LDL. Seu mecanismo de ação está relacionado a sua ligação a fatores de transcrição peroxisome proliferator-activated receptors (PPARs) e modulação de genes envolvidos no metabolismo lipídico. A lipoproteína lipase, responsável pela hidrólise de triglicerídeos, tem sua expressão aumentada, enquanto que a apolipoproteína C-III, um inibidor da lipoproteína lipase, tem sua expressão diminuída. Além disso, os fibratos diminuem a produção de apolipoproteína B e VLDLs ${ }^{11}$.

Os principais fármacos comercializados desta classe são o clofibrato, o bezafibrato, o etofibrato, o fenofibrato, o genfibrosil, o ciprofibrato. A redução dos níveis lipídicos observada com estes fármacos é de 10-25\% para níveis de colesterol e 20-40\% para níveis de triglicerídeos. Os níveis de HDL-c tem um aumento de $10-25 \%{ }^{11}$.

\subsubsection{Farmacogenética dos fibratos}

Existem vários genes de interesse que são alvos dos PPARs que podem interferir na eficácia dos fibratos, como a lipoproteína lípase, apolipoproteína CIII, apolipoproteína A-I, entre outros. No entanto poucos estudos farmacogenéticos avaliaram a influência de variantes nestes genes sobre a resposta ao trata- mento com fibratos. Em pacientes que utilizam genfibrozil, portadores do alelo N9 do gene da LPL apresentaram níveis maiores de subfrações mais aterogênicas de LDL quando comparados a não portadores ${ }^{56}$.

Em relação ao próprio gene $\operatorname{PPAR} \alpha$, no estudo SENDCAP conduzido com pacientes que utilizaram bezafibrato, portadores da variante $162 \mathrm{~V}$ apresentaram uma redução duas vezes maior de colesterol total quando comparados aos homozigotos $162 \mathrm{~L}^{57}$. Foucher et al. ${ }^{58}$ analisaram a influência de variantes em 5 genes candidatos (CETP, LPL, LIPC, PPAR $\alpha$ e APOE) sobre a resposta do tratamento com fenofibrato 200 $\mathrm{mg}$ ao dia e verificaram que a variante $\mathrm{G} / \mathrm{C}$ no intron 7 do gene PPARa e a variante S447X do gene $L P L$ são importantes preditores para a resposta dos níveis de triglicerídeos. Os resultados relacionados ao gene da apolipoproteína E são conflitantes. Enquanto alguns estudos não demonstram nenhuma influência deste gene $^{20,59}$ outros estudos mostram que a resposta, assim como para o tratamento com estatinas, é menor em portadores do alelo $E^{* 4}{ }^{60}$. Segundo alguns autores, o efeito do genótipo da apo E pode ser dependente do tipo de desordem lipídica do paciente ${ }^{61}$.

\section{3- Outros fármacos hipolipemiantes}

As resinas seqüestrantes de ácidos biliares (colestiramina e colestipol) se ligam aos ácidos biliares no lumen intestinal, impedindo sua reabsorção. O efeito hipolipemiante desta classe de fármacos está associada à expressão aumentada da enzima colesterol $7 \alpha$ hidroxilase (CYP7), enzima chave na rota de síntese de ácidos biliares. O efeito hipolipemiante é observado principalmente sobre os níveis de colesterol (redução de até 30\%). Os níveis de triglicerídeos podem aumentar com o tratamento ${ }^{11}$.

A niacina é um fármaco hipolipemiante bastante antigo cujo mecanismo de ação está relacionado à diminuição da liberação de ácidos graxos dos adipócitos, o que diminui a produção de VLDL. O resultado observado é uma acentuada diminuição dos níveis de triglicerídeos (ate -45\%) e de colesterol (-20\%) com aumento concomitante dos níveis de HDL-c ${ }^{11,16}$.

O probucol é um fármaco antioxidante, com propriedades hipolipemiantes que atua diminuindo os níveis de LDL-c e HDL-c, sem afetar os níveis de triglicerídeos. A diminuição dos níveis de HDL-c observada é decorrente do aumento da atividade da CETP, proteína transferidora de ésteres de colesterol ${ }^{11,16}$. 
A pesquisa farmacogenética relacionada a estes fármacos ainda é incipiente. Embora inúmeros genes de interesse possam ser pesquisados, os resultados estão voltados principalmente para a apolipoproteína E. Para o probucol, por exemplo, portadores do alelo $E^{* 4}$ parecem se beneficiar mais do tratamento hipolipemiante do que homozigotos $E^{*} 3 / E^{*} 3$ ou portadores do alelo $E^{* 2}{ }^{62}$, justamente o inverso do observado para o tratamento com estatinas.

\section{4- Relevância clínica e perspectivas futuras}

Quando o assunto é fármacogenética, o que se espera é que os resultados observados nos estudos possam ser utilizados para o aumento da eficácia do tratamento, mas será que as diferenças observadas na resposta têm significado clínico? Uma das principais limitações da farmacogenética hoje é que ela é estritamente experimental, baseada na correlação gene-resposta estatisticamente e não verificável ao nível individual na prática. No entanto, se as diferenças observadas entre genótipos para os níveis de HDL-c e LDL-c forem confirmadas individualmente, certamente estes achados terão aplicação prática. Dados dos estudos com hipolipemiantes mostram que o risco de eventos cardiovasculares pode ser diminuído $1 \%$ para cada $1 \%$ da redução dos níveis de LDL-c ${ }^{63}$ e 2 a $3 \%$ para cada $1 \mathrm{mg}$ de aumento dos níveis de HDL-c ${ }^{64}$. Isto sugere que uma diferença entre genótipos de apenas 5-7\% na resposta dos níveis de LDL-c, como a observada por Fiegenbaum et al. ${ }^{39}$, e de $13 \%$ nos níveis de HDL-c, como a observada pelos mesmos pesquisadores para o gene CETP ${ }^{23}$, já pode ser considerada biologicamente importante, principalmente entre os pacientes de maior risco.

Em um estudo realizado com 4888 pacientes, envolvendo as cinco estatinas presentes no mercado, foi observado que somente $38 \%$ dos pacientes atingiram as metas de LDL-c, segundo as diretrizes americanas ${ }^{65}$. Segundo os pesquisadores, o principal motivo do "insucesso" do tratamento foi à utilização de doses inadequadas dos fármacos. A utilização da fármacogenética, neste caso, poderia identificar aqueles pacientes que tem uma resposta reduzida e um ajuste de dose poderia fazê-los atingir as metas de LDL-c.

Um dos principais benefícios da implementação da farmacogenética, sem dúvida, será a redução de efeitos adversos aos medicamentos. Em uma metaanálise bastante citada em artigos publicados na área, Lazarou et al. ${ }^{66}$ verificaram que reações adversas a medicamentos (ADRs) constituem a $5^{\circ}$ causa de mortalidade nos Estados Unidos. A cada ano 2,1 milhões de prescrições resultam em efeitos adversos e destas cerca de um milhão resulta em hospitalização e mais de 100.000 pacientes evoluem para o óbito.

O conceito de uma terapia individualizada, onde o "fármaco certo é administrado na dose correta para o paciente correto" também é bastante promissor, pois a redução dos efeitos adversos além de ser alcançada pela seleção do melhor fármaco e a melhor dose para aqueles pacientes que tem metabolização deficiente, poderá ainda abrir as portas para a pesquisa e o desenvolvimento de novos alvos terapêuticos, que não sejam metabolizados por determinada enzima ou via.

Embora teoricamente as metas da farmacogenética dos efeitos adversos sejam promissoras, na prática, sua aplicação ainda não é tão clara. Nesta revisão foram apresentados alguns resultados de associação entre variantes e predisposição a efeitos adversos bastante comum com a utilização de inibidores da HMG Coa redutase. Utilizando os resultados de Fiegenbaum et al. ${ }^{39}$ para a variante $3435 \mathrm{C}>\mathrm{T}$ como exemplo, observa-se que o alelo $\mathrm{C}$ é mais freqüente (67\%) entre aqueles que desenvolveram mialgia durante o tratamento do que naqueles que não desenvolveram este efeito adverso (44\%). No entanto, estes resultados não podem ser utilizados diretamente para predição de efeitos adversos, uma vez que o valor preditivo seria muito baixo. Estes resultados, se confirmados posteriormente, poderão ser úteis se outras variantes associadas ao desenvolvimento de efeitos adversos forem descobertas e aí sim, definir subgrupos para os quais o medicamento não é recomendado.

Os resultados acumulados até o momento são bastante encorajadores e sugerem que o conceito do "fármaco certo para o paciente correto" pode se tornar realidade. A perspectiva é a de que, nas próximas décadas, ocorra a identificação de grande parte da variabilidade genética responsável pelas diferenças interindividuais na ação de fármacos e de que inúmeros novos exemplos de associação genoma-resposta a fármacos sejam compilados. O desafio a partir daí, será como e quando teremos isto como prática da medicina. 
Fiegenbaum M, Hutz MH. Pharmacogenetic of lipid-lowering drugs. Medicina (Ribeirão Preto) 2006; 39 (4): 543-53.

ABSTRACT: Although, the pharmacological treatment for cholesterol reduction has been an advance in the cardiovascular and atherosclerosis treatment, the benefits are still limited due to interindividual variability in the response to these drugs. Disease severity, treatment adhesion, physiological conditions, biological conditions as well as the patient genetic profile could be cited as important factors for the evaluation of interindividual variability. In this context, three large groups of genes could be investigated: a) Genes that codify proteins involved in metabolism and/ or transport of drugs, influencing the pharmacokinetics of these compounds. b) Genes that codify proteins involved in the mechanism of action and/or in the metabolic pathway where of drug action (pharmacodynamics). c) Genes that codify proteins involved in the direct development of the disease or in intermediate phenotypes. In this revision we discussed pharmacogenetic studies of the main lipid lowering drugs, and the expectations in relation to pharmacogenetics in order to help our prediction capacity in relation to treatment efficacy as well as the possibility to reduce adverse effects of these drugs. As soon as new studies would be performed and that most of the genetic variability associated with drug action would be disclosed, the great challenge would be the application of this knowledge in practical medicine.

Keywords: Lipid Lowering Drugs. Pharmacogenetics. Variation (Genetics). Cardiovascular Diseases.

\section{REFERÊNCIAS}

1 - Tunstall-Pedoe H, Vanuzzo D, Hobbs M, Mähönen M, Cepaitis $Z$, Kuulasmaa $\mathrm{K}$ et al. Estimation of contribution of changes in coronary care to improving survival, event rates, and coronary heart disease mortality across the WHO MONICA Project populations. Lancet 2000; 355: 688-700.

2 - Mansur AP, Favarato D, Souza MFF, Avakian SD, Aldrighi JM, Cesar LAM, Ramires JA. Trends in death from circulatory diseases in Brazil between 1979 and 1996. Arq Bras Cardiol 2001; 76: 504-10.

3 - Santos RD. III Diretrizes Brasileiras Sobre Dislipidemias e Diretriz de Prevenção da Aterosclerose do Departamento de Aterosclerose da Sociedade Brasileira de Cardiologia. Arq Bras Cardiol 2001; 77(Supl III): 1-48.

4 - National Cholesterol Education Program. Executive summary of the third report of the national cholesterol education program (NCEP). Expert panel on detection, evaluation and treatment of high blood cholesterol in adults (Adult treatment panel III). JAMA 2001; 285: 2486-96.

5 - Sacks FM, Pfeffer MA, Moye LA, Rouleau JL, Rutherford JD, Cole TG, Brown L, Warnica JW, Arnold JM, Wun CC, Davis BR, Braunwald $E$. The effect of pravastatin on coronary events after myocardial infarction in patients with average cholesterol levels. Cholesterol and Recurrent Events Trial investigators. N Engl J Med 1996; 335:1001-9.

6 - Miettinen TA, Gylling H, Strandberg T, Sarna S. Baseline serum cholestanol as predictor of recurrent coronary events in subgroup of Scandinavian simvastatin survival study. Finnish 4S Investigators. BMJ 1998; 316:1127-30.

7 - Hoffmann MM, Winkelmann BR, Wieland H, März W. The signifance of genetic polymorphisms in modulating the response to lipid-lowering drugs. Pharmacogenomics 2001; 2: 1-10.
8 - Kajinami K, Takekoshi N, Brousseau ME, Schaefer EJ. Pharmacogenetics of HMG-Coa reductase inhibitors: exploring the potential for genotype-based individualization of coronary heart disease management. Atherosclerosis 2004; 177: 219-34.

9 - Goldstein JL, Brown MS. Regulation of the mevalonate pathway. Nature 1990; 343: 425-30.

10 - Christians U, Jacobsen W, Floren LC. Metabolism and drug interactions of 3-hydroxy-3-methylglutaryl coenzyme A reductase inhibitors in transplant patients: are the statins mechanistically similar? Pharmacol Ther 1998; 80: 1-34.

11 - Bhatnagar D. Lipid-lowering drugs in the management of hyperlipidaemia. Pharmacol Ther 1998; 79: 205-30.

12 - Gotto AM. Statin therapy: where are we? Where do we go next? Am J Cardiol 2001; 87(Suppl): 13B-8B.

13 - Waters DD. Are we aggressive enough in lowering cholesterol? Am J Cardiol 2001; 88 (Suppl): 10F-5F.

14 - Kreisberg RA, Oberman A. Lipids and atherosclerosis: lessons learned from randomized controled trials of lipid lowering and other relevant studies. J Clin Endocrionol Metab 2002; 87: 423-37.

15 - Mahley RW. Apolipoprotein E: cholesterol transport protein with expanding role in cell biology. Science 1988; 240 : 62230.

16 - Mahley RW, Rall Jr SC. Apolipoprotein E: far more than a lipid transport protein. Annu Rev Gen Hum Gen 2000; 1: 507-37.

17 - Ordovas JM, Lopez-Miranda J, Perez-Jimenez F, Rodriguez C, Park JS, Cole T, Schaefer EJ. Effect of apolipoprotein E and A-IV phenotypes on the low density lipoprotein response to HMG CoA reductase inhibitor therapy. Atherosclerosis 1995; 113: 157-66. 
18 - Ballantyne CM, Herd JA, Stein EA, Ferlic LL, Dunn JK, Gotto AM Jr, Marian AJ. Apolipoprotein E genotypes and response of plasma lipids and progression-regression of coronary atherosclerosis to lipid-lowering drug therapy. J Am Coll Cardiol 2000; 36: 1572-8.

19 - Pedro-Botet J, Schaefer EJ, Bakker-Arkema RG, Black DM, Stein EM, Corella D, Ordovas JM. Apolipoprotein E genotype affects plasma lipid response to atorvastatin in a gender specific manner. Atherosclerosis 2001; 158: 183-93.

20 - Sanllehy C, Casals E, Rodriguez-Villar C, Zambon D, Ojuel J, Ballesta AM, Ros E. Lack of interaction of apolipoprotein E phenotype with the lipoprotein response to lovastatin or gemfibrozil in patients with primary hypercholesterolemia. Metabolism 1998; 47: 560-5.

21 - Pena R, Lahoz C, Mostaza JM, Jimenez J, Subirats E, Pinto X Taboada M, Lopez-Pastor A; Rap Study Group. Effect of apoE genotype on the hypolipidaemic response to pravastatin in an out patient stetting. J Intern Med 2002; 251: 518-25.

22 - Chasman DI, Posada D, Subrahmanyan L, Cook NR, Stanton VP, Ridker PM. Pharmacogenetic study of statin therapy and cholesterol reduction. JAMA 2004; 291: 2821-7.

23 - Fiegenbaum M, da Silveira FR, Van der Sand CR, Van der Sand LC, Ferreira ME, Pires RC, HutzMH. Pharmacogenetic study of apolipoprotein E, cholesteryl ester transfer protein and hepatic lipase genes and simvastatin therapy in Brazilian subjects. Clin Chim Acta. 2005; 362:182-8.

24 - Hannuksela ML, Liinamaa MJ, Kesaniemi YA, Savolainen MJ. Relation of polymorphisms in the cholesteryl ester transfer protein gene to transfer activity and plasma lipoprotein levels in alcohol drinkers. Atherosclerosis 1994; 110: 35-44.

25 - Kuivenhoven JA, de Knijff P, Boer JM, Smalheer HA, Botma GJ, Seidell JC, Kastelein JJ, Pritchard PH. Heterogeneity at the CETP gene locus: influence on plasma CETP concentrations and HDL cholesterol levels. Arterioscler Thromb Vasc Biol 1997; 17: 560-8

26 - Kuivenhoven JA, Jukema JW, Zwinderman AH, de Knijff $P$, McPherson R, Bruschke AVG, Lie KI, Kastelein JJ. The role of a common variant of the cholesteryl ester transfer protein gene in the progression of coronary atherosclerosis. $\mathrm{N}$ Engl J Med 1998; 338: 86-93

27 - Boekholdt SM, Thompson JF. Natural genetic variation as a tool in understanding the role of CETP in lipid levels and disease. J Lipid Res 2003; 44: 1080-93.

28 - Carlquist JF, Muhlestein JB, Horne BD, Hart NI, Bair TL, Molhuizen HO, Anderson JL. The cholesteryl ester transfer protein Taq1B gene polymorphism predicts clinical benefit of statin therapy in patients with significant coronary artery disease. Am Heart J 2003; 146:1007-14.

29 - Winkelmann BR, Hoffmann MM, Nauck M, Kumar AM, Nandabalan K, Judson RS, Boehm BO, Tall AR, Ruano G, Marz W.. Haplotypes of the Cholesteryl ester transfer protein predict lipid-modifying response to statin therapy. Pharmacogenomics J 2003; 3: 284-96.

30 - van Venrooij FV, Stolk RP, Banga JD, Sijmonsma TP, van Tol A, Erkelens DW, Dallinga-Thie GM; DALI Study Group. Common cholesteryl ester transfer protein gene polymorphisms and the effect of atorvastatin therapy in type 2 diabetes. Diabetes Care 2003; 26: 1216-23.
31 - Eberle D, Hegarty B, Bossard P, Ferre P, Foufelle F. SREBP transcription factors: master regulators of lipid homeostasis. Biochimie 2004; 86: 839-48.

32 - Brown MS, Goldstein JL. A proteolytic pathway that controls the cholesterol content of membranes, cells, and blood. Proc Natl Acad Sci USA 1999; 96: 11041-8.

33 - Fiegenbaum M, Silveira FR, Van der Sand CR, Van der Sand LC, Ferreira ME, Pires RC, Hutz MH. Determinants of variable response to simvastatin treatment: the role of common variants of SCAP, SREBF-1a and SREBF-2 genes. Pharmacogenomics J 2005; 5: 359-64.

34 - Kajinami K, Brousseau ME, Ordovas JM, Schaefer EJ. Interactions between common genetic polymorphisms in ABCG5/ G8 and CYP7A1 on LDL cholesterol-lowering response to atorvastatin. Atherosclerosis 2004; 175:287-93.

35 - Igel M, Sudhop T, von Bergmann K. Metabolism and drug interactions of 3-hydroxy-3-methylglutaryl-coenzyme A-reductase inhibitors (statins). Eur J Clin Pharmacol 2001; 57: 357-64.

36 - Lamba JK, Lin YS, Schuetz EG, Thummel KE. Genetic contribution to variable human CYP3A-mediated metabolism. Adv Drug Deliv Rev 2002; 54: 1271-94.

37 - Kajinami K, Brousseau ME, Ordovas JM, Schaefer EJ. CYP3A4 genotypes and plasma lipoprotein levels before and after treatment with atorvastatin in primary hypercholesterolemia. Am J Cardiol 2004; 93: 104-7.

38 - Kivisto K, Niemi M, Schaefeller E, Pitkala K, Tilvis R, Fromm MF, Schwab M, Eichelbaum M, Strandberg T. Lipid-lowering response to statins is affected by CYP3A5 polymorphism. Pharmacogenetics 2004 ; 14 : 523-5.

39 - Fiegenbaum M, da Silveira FR, Van der Sand CR, Van der Sand LC, Ferreira ME, Pires RC, Hutz MH. The role of common variants of $A B C B 1, C Y P 3 A 4$, and CYP3A5 genes in lipid-lowering efficacy and safety of simvastatin treatment. Clin Pharmacol Ther 2005; 78: 551-8.

40 - Ho RH. Kim, RB. Transporters and drug therapy: implications for drug disposition and disease. Clin Pharmacol Ther 2005; 78: $260-77$.

41 - Hoffmeyer S, Burk O, von Richter O, Arnold HP, Brockmöller J, Johne A, Cascorbi I, Gerloff T, Roots I, Eichelbaum M, Brinkmann U.. Functional polymorphisms of the human multidrug-resistance gene: multiple sequence variations and correlation of one allele with P-glycoprotein expression and activity in vivo. Proc Natl Acad Sci USA 2000; 97: 3473-8.

42 - Cascorbi I, Gerloff T, Johne A, Meisel C, Hoffmeyer S, Schwab M, Schaeffeler E, Eichelbaum M, Brinkmann U, Roots I. Frequency of single nucleotide polymorphisms in the P-glycoprotein drug transporter MDR1 gene in white subjects. Clin Pharmacol Ther 2001; 69: 169-74.

43 - Schaeffeler E, Eichelbaum M, Brinkmann U, Penger A, AsantePoku S, Zanger UM, Schwab M. Frequency of C3435T polymorphism of MDR1 gene in African people. Lancet 2001; 358: $383-4$

44 - Kajinami K, Brousseau ME, Ordovas JM, Schaefer EJ. Polymorphisms in the multidrug resistance-1 (MDR1) gene influence the response to atorvastatin treatment in a genderspecific manner. Am J Cardiol 2004; 93: 1046-50. 
45 - Thompson JF, Man M, Johnson KJ, Wood LS, Lira ME, Lloyd DB, Banerjee P, Milos PM, Myrand SP, Paulauskis J, Milad MA, Sasiela WJ.. An association study of 43 SNPs in 16 candidate genes with atorvastatin response. Pharmacogenomics J 2005; 5: 352-8.

46 - Rodrigues AC, Rebecchi IM, Bertolami MC, Faludi AA, Hirata $\mathrm{MH}$, Hirata RD. High baseline serum total and LDL cholesterol levels are associated with MDR1 haplotypes in Brazilian hypercholesterolemic individuals of European descent. Braz J Med Biol Res 2005; 38: 1389-97.

47 - Tamai I, Nezu J. Uchino H, Sai Y, Oku A, Shimane M, Tsuji A. Molecular identification and characterization of novel members of the human organic anion transporter (OATP) family. Biochem Biophys Res Commun 2000; 273: 351-60.

48 - Kullak-Ublick GA, Ismair MG, Stieger B, Landmann L, Huber R, Pizzagalli F, Fattinger K, Meier PJ, Hagenbuch B...Organic anion-transporting polypeptide B (OATP-B) and functional comparison with three others OATPs of human liver. Gastroenterology 2001; 120: 525-33.

49 - Mwinyi J, Johne A, Bauer S, Roots I, Gerloff T. Evidence for inverse effects of OATP-C (SLC21A6) 5 and 1b haplotypes on pravastatin kinetics. Clin Pharmacol Ther 2004; 75: 41521.

50 - KIM RB. 3-hidroxy-3-methylglutaryl-coenzyme A reductase inhibitors (statins) and genetic variability (single nucleotide polymorphisms) in a hepatic drug uptake transporter: what's it all about? Clin Pharmacol Ther 2004; 75: 381-5.

51 - Nishizato Y, leiri I, Suzuki H, Kimura M, Kawabata K, Hirota T, Takane H, Irie S, Kusuhara H, Urasaki Y, Urae A, Higuchi S, Otsubo K, Sugiyama Y. Polymorphisms of OATP-C (SLC21A6) and OAT3 (SLC22A8) genes: consequences for pravastatin pharmacokinetics. Clin Pharmacol Ther 2003; 73: 554-65.

52 - Omar MA, Wilson JP, Cox TS. Rhabdomyolysis and HMGCoa reductase inhibitors. Ann Pharmacother 2001; 35:10961107.

53 - Ballantyne CM, Corsini A, Davidson MH, Holdaas H, Jacobson TA, Leitersdorf E, Marz W, Reckless JP, Stein EA. Risk for myopathy with statin therapy in high-risk patients. Arch Intern Med 2003; 163: 553-64.

54 - Hamilton-Craig I. Statin-associated myopathy. Med J Aust 2001; 175: 486-9.

55 - Wilke RA, Moore JH, Burmester JK. Relative impact of CYP3A genotype and concomitant medication on the severity of atorvastatin-induced muscle damage. Pharmacogenet Genomics 2005; 15: 415-21.

56 - Brousseau ME, Goldkamp AL, Collins D, Demissie S, Connolly AC, Cupples LA, Ordovas JM, Bloomfield HE, Robins SJ, Schaefer EJ.. Polymorphisms in the gene encoding lipoprotein lipase in men with low HDL-C and coronary heart disease: the Veterans Affairs HDL Intervention Trial. J Lipid Res 2004; 45: 1885-91.
57 - Flavell DM, Pineda Torra I, Jamshidi Y, Evans D, Diamond JR, Elkeles RS, Bujac SR, Miller G, Talmud PJ, Staels B, Humphries SE.. Variation in the PPAR alpha gene is associated with altered function in vitro and plasma lipid concentrations in Type II diabetic subjects. Diabetologia 2000; 43: 673-80.

58 - Foucher C, Rattier S, Flavell DM, Talmud PJ, Humphries SE, Kastelein JJ, Ayyobi A, Pimstone S, Frohlich J, Ansquer JC, Steiner G._Response to micronized fenofibrate treatment is associated with the peroxisome-proliferator-activated receptors alpha G/C intron7 polymorphism in subjects with type 2 diabetes. Pharmacogenetics 2004; 14: 823-9.

59 - Manttari M, Koskinen P, Ehnholm C, Huttunen JK, Manninen V. Apolipoprotein $\mathrm{E}$ polymorphism influences the serum cholesterol response to dietary intervention. Metabolism 1991; 40: 217-21.

60 - Yamada M. Influence of apolipoprotein E polymorphism on bezafibrate treatment response in dyslipidemic patients. $\mathrm{J}$ Atheroscler Thromb 1997; 4: 40-4.

61 - Nemeth A, Szakmary K, Kramer J, Dinya E, Pados G, Fust G, Huettinger M. Apolipoprotein $E$ and complement $C 3$ polymorphism and their role in the response to gemfibrozil and low fat low cholesterol therapy. Eur J Clin Chem Clin Biochem 1995; 33: 799-804.

62 - Eto M, Sato T, Watanabe K, Iwashima Y, Makino I. Effects of probucol on plasma lipids and lipoproteins in familial hypercholesterolemic patients with and without apolipoprotein E4. Atherosclerosis 1990; 84: 49-53.

63 - Grundy SM, Cleeman JI, Merz NB, Brewer HB, Clark LT, Hunninghake DB, Pasternak RC, Smith Jr SC, Stone NJ. Implications of recent clinical trial for the National Cholesterol Education Program Adult Treatment Panel III guidelines. J Am Coll Cardiol 2004; 44: 720-32.

64 - Dean BB, Borenstein JE, Henning JM, Knight K, Merz NB. Can change in high-density lipoprotein cholesterol levels reduce cardiovascular risk? Am Heart J 2004; 147: 966-76.

65 - Pearson T, Laurora I, Chu H, Kafonek S. The lipid treatment assessment project (L-TAP). Arch Intern Med 2000; 160: 459-67.

66 - Lazarou J, Pomeranz BH, Corey PN. Incidence of adverse drug reactions in hospitalized patients: a meta-analysis of prospective studies. JAMA 1998; 279: 1200-5.

67 - Freeman DJ, Samani NJ, Wilson V, McMahon AD, Braund PS, Cheng S, Caslake MJ, Packard CJ, Gaffney D._A polymorphism of the cholesteryl ester transfer protein gene predicts cardiovascular events in non-smokers in the West of Scotland Coronary Prevention Study. Eur Heart J 2003; 24:1833-42.

68 - Salek L, Lutucuta S, Ballantyne CM, Gotto Jr AM, Marian AJ. Effects of SREBF-1a and SCAP polymorphisms on plasma levels of lipids, severity, progression and regression of coronary atherosclerosis and response to therapy with fluvastatin. J Mol Med 2002; 80: 737-44. 